\title{
Research on a Novel Model of Virus Spreading in the Short Message Network
}

\author{
Liwei XING \& Kunbao CAI \\ College of Communication Engineering, Chongqing University, Chongqing 400044, P. R. of China
}

\begin{abstract}
Based on investigating the classical models of epidemic spreading, a novel model of virus spreading in the short message network is presented. A set of state equations describing the nonlinear dynamic system of the presented model is established. The effect of individual model parameters on virus spreading characteristics is analyzed. The research results show that the presented model can better reveal the virus spreading characteristics in the short message network of mobile phones, which is valuable for researching on the prevention and treatment of mobile phone viruses.
\end{abstract}

KEYWORD: Short message virus; Virus spreading model; Nonlinear dynamic system; State equation

\section{INTRODUCTION}

With the advancement of the society informatization process, and user consumption ability enhancement, the degree of people's dependence on mobile phones and demand is increasing constantly. As reported on internet, the International Telecommunication Union predicted that at the end of 2014, the global mobile phone users will reach about 7 billion which is close to the global population, where more than $50 \%$ of the total number is from the Asia-Pacific Region. The rapid growth of the mobile phone users directly led to a surge of sent short messages. According to the report of the Ministry of Industry and Information Technology, in 2008 China's mobile phone short messages sent 699.67 billion articles. In 2009, China mobile's short messages reached 1.8 billion per day.

The rapid development of the short message service of mobile phones is of great significance to the development of national economy. At the same time, it has brought some new problems to users and service providers, such as spam messages, pornographic messages and wide spreading of the virus in the short message network that cause a lot of loss and troubles for both users and service providers. Due to the mobile phones have become a basic tool of people's daily life and work, and concern personal privacy, the dangerous degree of mobile phone virus in the aspect of information security may be more than a computer virus. It is no doubt that the study of the virus spreading characteristics in the short message network and the prevention and treatment of the virus have an important practical significance.

\section{RESEARCH STATUS}

Although the research on the short message virus has started for several years and caused a widespread attention, the spreading characteristics of the short message virus and the effective prevention and treatment are still at outset stage. However, the successful application of complex network theory to the research of epidemic and infectious disease spreading in human groups, as well as the research results about the virus spreading in computer networks provide new ideas and methods for the research of virus spreading in the short message network (Boguna et al. 2002, Pastor-Satorras et al 2001, Newman et al. 2002, Xu et al. 2004).

It is noted that the classical virus spreading models are SI, SIS and SIR models, where the characters S, I and R represent the Susceptible, Infected and Recovered states, respectively (Xu et al. 2004). Here, the recovered state represents that an infected individual has been cured by some measures, and thus has obtained the immunity against a kind of virus. These fundamental models are too simple to be successfully used in the short message network. For examples: the SI model does not consider that an infected individual can be cured by an effective measure; the SIS model does not think about that the cured individual may obtain the immunity against the virus; the SIR model does not consider such a situation that an recovered individual may be infected repeatedly. The authors of this paper have noted that a number of researchers presented some improved models: For examples: in an extended model denoted as SPIR (Jin et al. 2010), 
some parameters about the infection rate, virus immune rate and immune failure rate are introduced, and the priority connection characteristics in the network is also considered; an SEIR model for the virus spreading in the short message network was proposed (Chen et al. 2009), which has considered the small world characteristics of the network, and on this basis they studied the effect of user immune and virus mutation on the virus spreading in the short message network.

\section{VIRUS SPREADING CHARACTERISTICS}

Generally, the mobile phone virus spreads via the transmission and forwarding relay of short messages in the network. This is based on the topology of the network. It was verified that the short message network is a scale-free network whose node degree follows the power-law distribution (Ma et al. 2006, Sun et al. 2009, Wu et al. 2007). Generally, the virus spreading follows the properties below:

1) Only when the user opens the links in an infected short message or downloads and installs the attachment, the virus will be activated.

2) Most of the viruses are designed by aiming at the operating system vulnerabilities of mobile phones. Thus, if a user patches the system operating vulnerabilities in a timely manner, his or her mobile phone can be immune to these viruses.

3) Because some viruses may take place mutation, it is not possible for mobile phones to keep immunity to such viruses always.

4) The poisoning risk of mobile phones is closely related to the safety awareness of mobile phone users. This is different from one person to other one.

\section{NOVEL MODEL OF VIRUS SPREADING}

Based on the investigation of the existing models for the virus spreading in the short message network, the authors of this paper present a novel virus spreading model which is more approaching the realistic situation. The individual states of the users and the relations between state transitions are shown in the Figure 1. This model can be viewed as an extension of the SEIR model, where the meanings of the characters $\mathrm{S}, \mathrm{I}$ and $\mathrm{R}$ are the same as previous definitions, while the character $\mathrm{E}$ represents Exposed, that is, the user is at a latent period to be infected. As shown in Figure 1, the transition relations between individual states are as follows:

$\mathrm{S} \rightarrow \mathrm{E}$ : Represents that some users originally in the susceptible state in the network have received a short message carrying a virus, but the virus has not yet been activated. The state transition probability $p_{1}$ is the virus spreading rate.

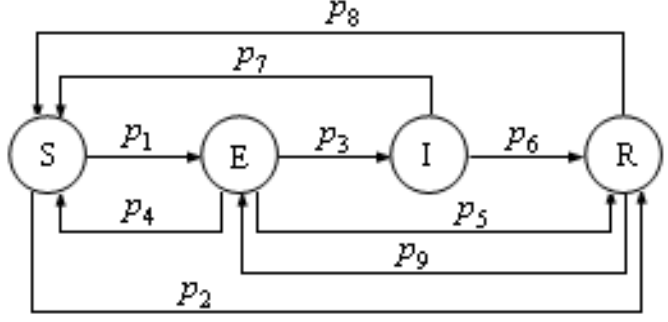

Figure 1. State transition diagram for the virus spreading model in the short message network.

$\mathrm{S} \rightarrow \mathrm{R}$ : Says that some of users in the susceptible state patched the operating system vulnerabilities in time, and thus their mobile phones have obtained the immunity to the virus. Here, $p_{2}$ is the transition probability from the susceptible state to the recovered state.

$\mathrm{E} \rightarrow \mathrm{I}$ : Indicates that due to the links in an infected short message were opened or the attachment was downloaded and installed, the virus was activated, and thus the users have caught the virus. Here, the state transition coefficient $p_{3}$ is the virus-infected rate.

$\mathrm{E} \rightarrow \mathrm{S}$ : Expresses that before the virus is activated, it has been deleted. Here, $p_{4}$ is the transition probability from the exposed state to the susceptible state.

$\mathrm{E} \rightarrow \mathrm{R}$ : Indicates that before the virus is activated, it has been deleted and the user has made a patch, and thus the mobile phone has obtained immune ability to the virus. Where $p_{5}$ is the transition probability from the exposed state to the recovered state.

$\mathrm{I} \rightarrow \mathrm{R}$ : Means that after being infected by a virus, some users have cleared the virus by some measures and patched successfully, and thus the mobile phones have obtained an immunizing ability to the virus. Here, $p_{6}$ is the transition probability from the infected state to the recovered state.

$\mathrm{I} \rightarrow \mathrm{S}$ : Says that some users have cleared a kind of virus through some means, but failed to patch in time. Where $p_{7}$ is the transition probability from the infected state to the susceptible state?

$\mathrm{R} \rightarrow \mathrm{S}$ : Represents that a virus has taken place a mutation and the users failed to timely patching, and thus the mobile phones originally in the immune state will transfer to the susceptible state again with a transition probability $p_{8}$.

$\mathrm{R} \rightarrow \mathrm{E}$ : Expresses that some mobile phones have received a short message carrying a virus, which makes these users in the recovered state transfer into the exposed state with a transition probability $p_{9}$.

Assume that at a time instant the percentages of the users in states of S、E、I and R occupying the total number of users in the short message network are, respectively, denoted by $s(t) 、 e(t) 、 i(t)$ and $r(t)$. Then, that leads to

$$
s(t)+e(t)+i(t)+r(t)=1
$$


If the total number of the users in the short message network is denoted by $N$, then the number of susceptible, exposed, infected and immune users can be represented as $N s(t), N e(t), N i(t)$ and $N r(t)$, respectively. Considering that each infected user can averagely infect $p_{1} \times s(t)$ susceptible users in a unit time, there are $N i(t) \times p_{1} \times s(t)=p_{1} \times N s(t) i(t)$ susceptible users that will transfer into the exposed users within the unit time. In the other hand, $p_{2} \times N s(t)$ susceptible users will transfer into immune users, and $p_{4} \times N e(t)$ exposed users, $p_{7} \times N i(t)$ infected users and $p_{8} \times N r(t)$ immune users will transfer into susceptible users. Thus, the change rate of the number of susceptible users is given by

$$
\begin{aligned}
N \frac{d s(t)}{d t}= & -p_{1} \times N s(t) i(t)-p_{2} \times N s(t)+ \\
& p_{4} \times N e(t)+p_{7} \times N i(t)+p_{8} \times N r(t)
\end{aligned}
$$

Similarly, the change rates of the exposed, infected and immune users can be, respectively, given by

$$
\begin{aligned}
N \frac{d e(t)}{d t}= & p_{1} \times N s(t) i(t)+p_{9} \times N r(t)- \\
& p_{3} \times N e(t)-p_{4} \times N e(t)-p_{5} \times N e(t) \\
N \frac{d i(t)}{d t}= & p_{3} \times N e(t)-p_{6} \times N i(t)-p_{7} \times N i(t) \\
N \frac{d r(t)}{d t}= & p_{2} \times N s(t)+p_{5} \times N e(t)+ \\
p_{6} & \times N i(t)-p_{8} \times N r(t)-p_{9} \times N r(t)
\end{aligned}
$$

Thus, a set of state equations, describing the virus spreading model in the short message network, can be represented as

$$
\begin{gathered}
\frac{d s(t)}{d t}=-p_{1} \times s(t) i(t)-p_{2} \times s(t)+ \\
p_{4} \times e(t)+p_{7} \times i(t)+p_{8} \times r(t) \\
\frac{d e(t)}{d t}=p_{1} \times s(t) i(t)+p_{9} \times r(t)- \\
p_{3} \times e(t)-p_{4} \times e(t)-p_{5} \times e(t) \\
\frac{d i(t)}{d t}=p_{3} \times e(t)-p_{6} \times i(t)-p_{7} \times i(t) \\
\frac{d r(t)}{d t}=p_{2} \times s(t)+p_{5} \times e(t)+ \\
p_{6} \times i(t)-p_{8} \times r(t)-p_{9} \times r(t)
\end{gathered}
$$

where $s(t) 、 e(t) 、 i(t)$ and $r(t)$ can be called as the state variables for the virus spreading model in the short message network. In fact, the set of state equations (6), (7), (8) and (9) mathematically describes a nonlinear dynamic system represented by the model in Figure 1.

\section{MODEL ANALYSIS}

To study the changing nature over time of the state variables in the nonlinear dynamic system, and thus to make an appropriate prevention method and strategy for the mobile phone virus, the nonlinear dynamic system is analyzed by using Matlab software as follows.

1) Assume that the initial conditions of the system are set as $s(0)=0.75, e(0)=0.1, i(0)=0.15$ and $r(0)=0$; the virus spreading rate is $p_{1}=0.01$; the transition probabilities between individual state variables are, respectively, given by $p_{2}=0.04$, $p_{3}=0.04, p_{4}=0.1, p_{5}=0.15, p_{6}=0.15$, $p_{7}=0.1$, and $p_{9}=0.01$. By solving the set of state equations (6), (7), (8) and (9), the changing nature over time of the state variables can be obtained that are shown in Figure 2. As indicated in Figure 2: since an immune measure is introduced in the model, the user percentages in susceptible, infected and exposed states are all decreasing with the increase of time; the user percentage in the immune state increases over time; with increasing time further, all state variables approaches individual steady state values, which indicates that most users will be in the immune state and only a small number of users will be in the susceptible state.

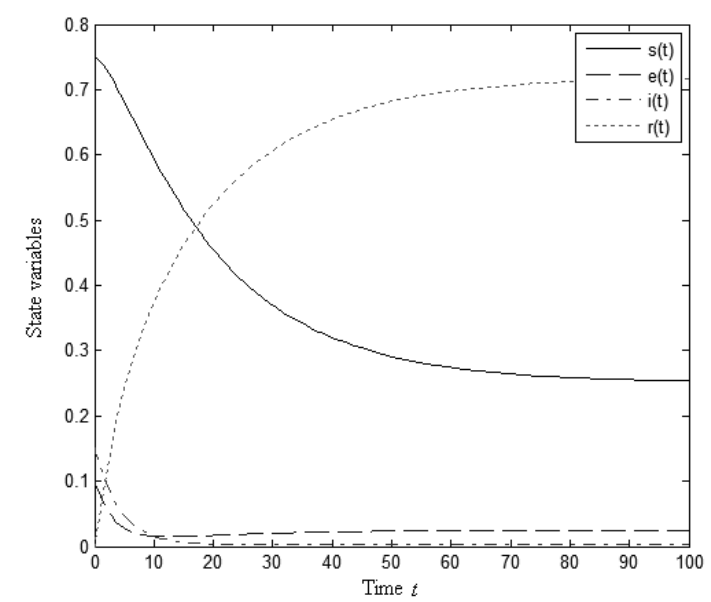

Figure 2. Changing nature over time of the state variables.

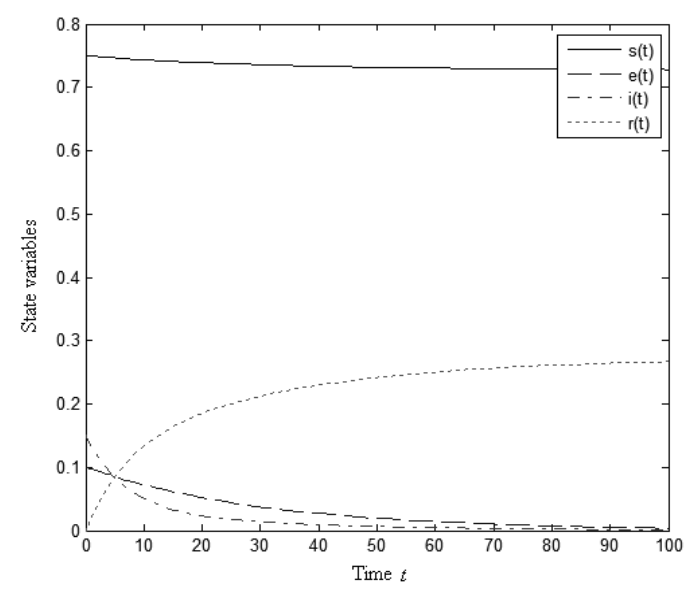

Figure 3. State variable responses for the SEIR model . 


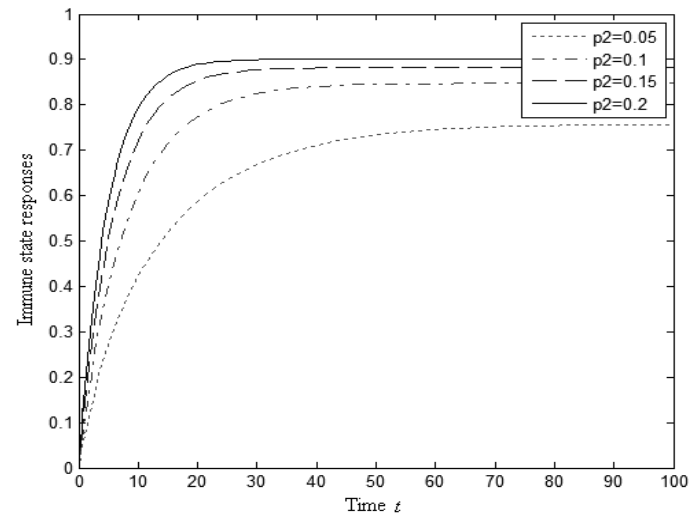

Figure 4. Immune state responses for different values of $p_{2}$.

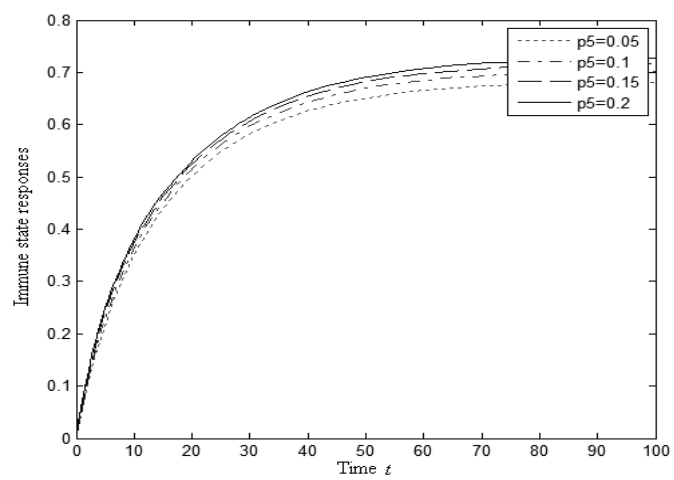

Figure 5. Immune state responses for different values of $p_{5}$.

2) If $p_{2}=p_{4}=p_{5}=p_{7}=p_{8}=p_{9}=0$, then the model presented in this paper degenerates to the conventional SEIR model. Furthermore, if the remainder state transition coefficients take the values as that for Figure 2, then the time responses of the individual state variables are shown in Figure 3. Comparing Figure 3 with Figure 2, it can be found that the system has almost reached the steady state at 100 time units. At this time, the percentages of the infected users in these two figures are all approaching zero, and the percentages of the exposed users in both figures are very small. The percentage of the susceptible users in the presented model is much lower than that in the SEIR model, and the percentage of the immune users in the presented model is much higher than that in the SEIR model. This is because of that: in the presented model, some users in the susceptible state put a patch and update a virus database in time, and thus their mobile phones obtain an immune ability to the virus; some exposed users have timely deleted the virus before it is to be activated and put a patch and update a virus database, and thus the mobile phones obtain an immune ability. These differences come from that in the presented model, the state transition probabilities $p_{2}$ and $p_{5}$ are unequal to zero.

3 ) Considering that a transition probability takes 4 different values, that is, $p_{2}=0.05, p_{2}=0.1$, $p_{2}=0.15$ and $p_{2}=0.2$, and the other transition probabilities are the same as that for Figure 2, the change of the immune state variable $r(t)$ over time is shown in Figure 4. From this figure, it can be seen that the greater the value of transition probability $p_{2}$, the greater the percentage of immune users will be.

4) Considering that the all transition probabilities take the same values as that for Figure 2, except for that $p_{5}$ takes 4 different values, i.e., $p_{5}=0.05$, $p_{5}=0.1, \quad p_{5}=0.15$ and $p_{5}=0.2$, the change of the percentages of the immune users over time is shown in Figure 5. Clearly, the greater the value of $p_{5}$ the greater the percentage of the immune users will be.

From Figures 4 and 5, it can be seen that the increase of the value of transition probabilities $p_{2}$ and $p_{5}$ can improve the inhibition ability of the virus spreading in the short message network.

\section{CONCLUSION}

Compared with the conventional virus spreading models, the main advantages of the model presented by this paper are that the effect of human intervention factors on the improvement of virus inhibition has been considered in this paper. For example, the users originally in the susceptible state can reduce the risk of virus infection by putting a patch and updating a virus database in time. The presented model has also considered that the virus mutation may lead to that some immune users transfer again to the susceptible or exposed state. Indeed, the presented model can better reflect the actual situation of the virus spreading in the short message network, and the study results have some practical significance to the virus prevention and comprehensive treatment.

\section{REFERENCES}

[1] Boguna, $\mathrm{M}$ et al. 2002. Epidemic spreading in correlated complex networks. Phys Rev E, 66, (4): 047104-047107.

[2] Chen, Xaiojiang. et al. 2009. Simulation research on mobile phone virus propagation model. Wuhan: Journal of Wuhan University of Technology, 31(1): 8-11.

[3] Jin, Songlin et al. 2010. Virus spreading model of mobile phones in the short message network. Computer Engineering and Applications, 46 (331):118-120.

[4] Ma, Baojun et al. 2006. Dynamics of continuous, discrete and impulsive systems. Evolution of the SMS Network, 13 (3-4):371-377.

[5] Newman, $M$ et al. 2002. Spread of epidemic disease on networks. Phys Rev E, 66(1): 016128-016139.

[6] Pastor-Satorras, R et al 2001. Epidemic spreading in scalefree networks. Physical Review Letters, 86(14): 32003203.

[7] Sun, Qindong et al.. 2009. A dynamic evolution model of short message complex network. Xian: Journal of Xian Jiaotong University, 43(6): 5-9.

[8] $\mathrm{Wu}$, Ye et al. 2007. Research on the growing process of short message networks. Beijing: Acta Physica Sinica, 56(4): 2037-2041.

[9] Xu, Dan et al. 2004. Application of complex network theory to the study of virus spreading on the Internet. Complex Systems and Complex Science, 1(3): 11-26. 